\title{
Insulator Detection and Defect Classification using Rotation Invariant Local Directional Pattern
}

\author{
Taskeed Jabid \\ Computer Science and Engineering Department \\ East West University \\ Dhaka, Bangladesh
}

\author{
Tanveer Ahsan \\ Computer Science and Engineering Department \\ International Islamic University Chittagong \\ Chittagong, Bangladesh
}

\begin{abstract}
Detecting power line insulator automatically and analyzing their defects are vital processes in maintaining power distribution systems. In this work, a rotation invariant texture pattern named rotation invariant local directional pattern (RILDP) is proposed for representing insulator image. For this at first, local directional pattern (LDP) is applied on image which can encode local texture pattern into an eight bit binary code by analyzing magnitude of edge response in eight different directions. Finally this LDP code is made robust to rotation by meticulously rearranging the generated another binary code which named as rotation invariant local directional pattern (RILDP). Insulator detection is carried out where this RI-LDP based histogram act as a feature vector and support vector machine (SVM) plays the role of the classifier. The detected insulator image region is further analyzed for possible defect identification. For this, an automatic extraction method of the individual insulator caps is proposed. The defect in segmented insulators is analyzed using LDP texture feature on individual cap region. We evaluated the proposed method using two sets of 493 real-world insulator images captured from a ground vehicle. The proposed insulator detector shows comparable performance to state-of-the-arts and our defect analysis method outperforms existing methods.
\end{abstract}

Keywords-Insulator detection; insulator defect analysis; local direction pattern (LDP); rotation invariant local directional pattern (RI-LDP); support vector machine (SVM)

\section{INTRODUCTION}

A moment without electricity cannot be imagined by our modern-day life. We, the citizen of modern-day society cannot imagine a moment without electricity. It is so much endemic to our every day exertion that the uninterrupted distribution of electricity is must. The possibility of uninterrupted distribution of electricity is associated with number of factors including problem free power line channel. That's why regular inspection and maintenance of power line distribution system is required. Among the number of components in power line distribution systems, insulator is one of the key components for stable power supply. According to the statistical data of the national power company, the breakdown of insulator is the most frequent cause of the failure of power system [1]. Therefore, rigorous insulator inspection for defect identification is necessary to ensure an uninterrupted power distribution. Until recently, the insulator inspection relies mostly on manual labor by climbing the pole which is dangerous and time-consuming [2]. However, advancement of vision based object detection technology promotes automatic insulator monitoring system which can detect the position of insulator and identify possible defect in the detected insulator [3] from captured images and/or videos.

A large number of insulator localization and defect identification works are done on aerial images and/or videos. Some of them utilize local image descriptor for recognizing insulators from clutter background. One of them is proposed by Oberweger et al. [4] where a circular descriptor generated by local gradient information. They utilize a unique voting scheme for accurate localization of insulator caps. On the other hand Liao and An [5] describe a local interest point by both gradient and gray level feature. They also utilize multiple circular image patches to describe a local interest point. This multiscale and multifeature (MSMF) descriptor is also able to handle some degree of rotation variation. Some researchers used characteristic shape features of insulators for the detection. In [6] insulator structure is modeled using Haar-like feature to enable rapid feature extraction. They generate a 3D insulator model which minimizes the scarcity of positive samples. Vertical projection curve is derived from video sequence by Bingfeng Li et al. [7] for SVM based insulator detection. Wang et al. [3] propose a Gabor based feature extraction method for insulator recognition. False positive which appears due to clutter background is discarded by morphological operation. Tiantian et al. [8] utilized local textured feature local binary pattern (LBP) and fuse it with histogram of oriented gradient (HOG) feature for insulator detection from aerial videos. Zhao et al [9] detect insulator using infrared images by taking advantage of high-level discriminative Convolution Neural Networks.

Even though there are a number of proposed systems for automatic insulator detection from aerial images, the high price and low stability make them less practical. Moreover, aerial vehicles are more susceptible to ill weather conditions (e.g., strong wind). Therefore, most of the systems utilize ground vehicles for insulator detection and subsequent defect analysis. Edge histogram descriptor (EHD) feature along with Kalman filter is used by Li et al. [1] to recognize the insulator. Jabid et al. proposed local textured based insulator detection [10]. A SVM based insulator condition analysis using wavelet transformation feature is proposed by Murthy et al. [11]. They also illustrate suitability of wavelet transformation based feature for identifying good insulator from bad ones using hidden Markov model in [12]. 
Developing a successful insulator monitoring system is a challenging problem due to the large variations of the appearance of insulator caused by scale, viewpoint, color, and occlusion (example insulator images are shown in Fig. 1). Cluttered backgrounds also increase the complexity of the problem and often increase the computational load and decrease the success rate in detection. Regarding the viewpoint, arbitrary in-plane and out-of-plane rotational angles make the detection problem highly challenging. Most of the existing insulator detection methods address only a subset of the variations without having the capability to handle all of them.
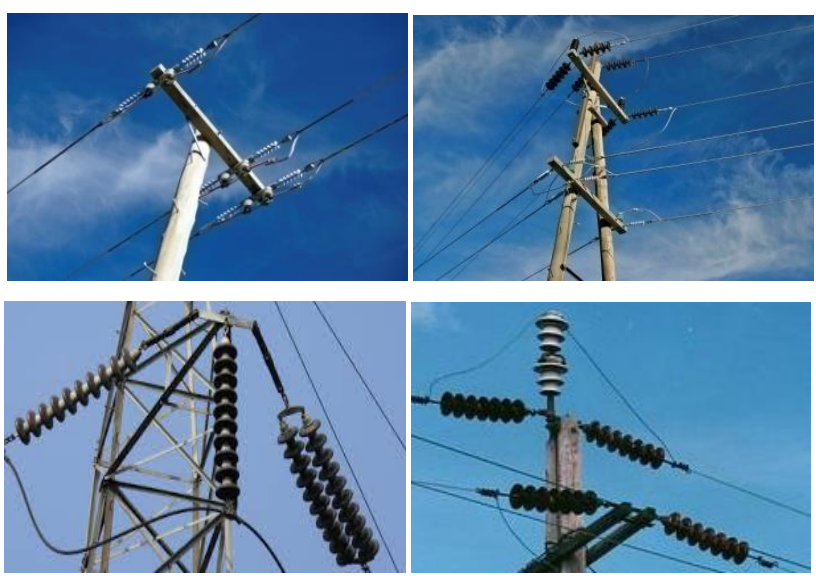

Fig. 1. Real life insulator appearance in different orientation.

In this work, an insulator monitoring system is proposed which can detect the insulators from images captured through the ground vehicle and subsequently analyze for potential defect. As the proposed insulator detection system utilizing a novel rotation invariant texture feature namely rotation invariant local directional pattern (RI-LDP), it can handle highly cluttered images with insulators appeared in arbitrary orientation. Image pyramid based multiscale detection approach is used to overcome the scale variation. The proposed method utilizes a novel rotation invariant texture encoding method to describe image which helps better detection of insulator region even if insulator appeared in arbitrary orientation.

The main strength of proposed encoding is two folds: firstly it describes local image texture by comparing relative strength of eight directional gradients. As gradient provides relatively robust information in adverse imaging situation, hence the proposed code inherently becomes more stable than other intensity based feature. Secondly, to make the feature robust to rotation, the code is rearranged according to direction of highest gradient direction. The effectiveness of the proposed RI-LDP is substantiated by the higher detection accuracy when classification is carried out utilizing support machine (SVM) classifier. Furthermore, we an automatic insulator defect analysis system is proposed which can automatically partition each cap of an insulator from its core and subsequently analyze each cap for the defect. Our defect analysis system can identify defected insulators and can categorize them into five common defects, i.e., Cracks, Contamination, Whitening, Bullet Damage, and Alligatoring effects.

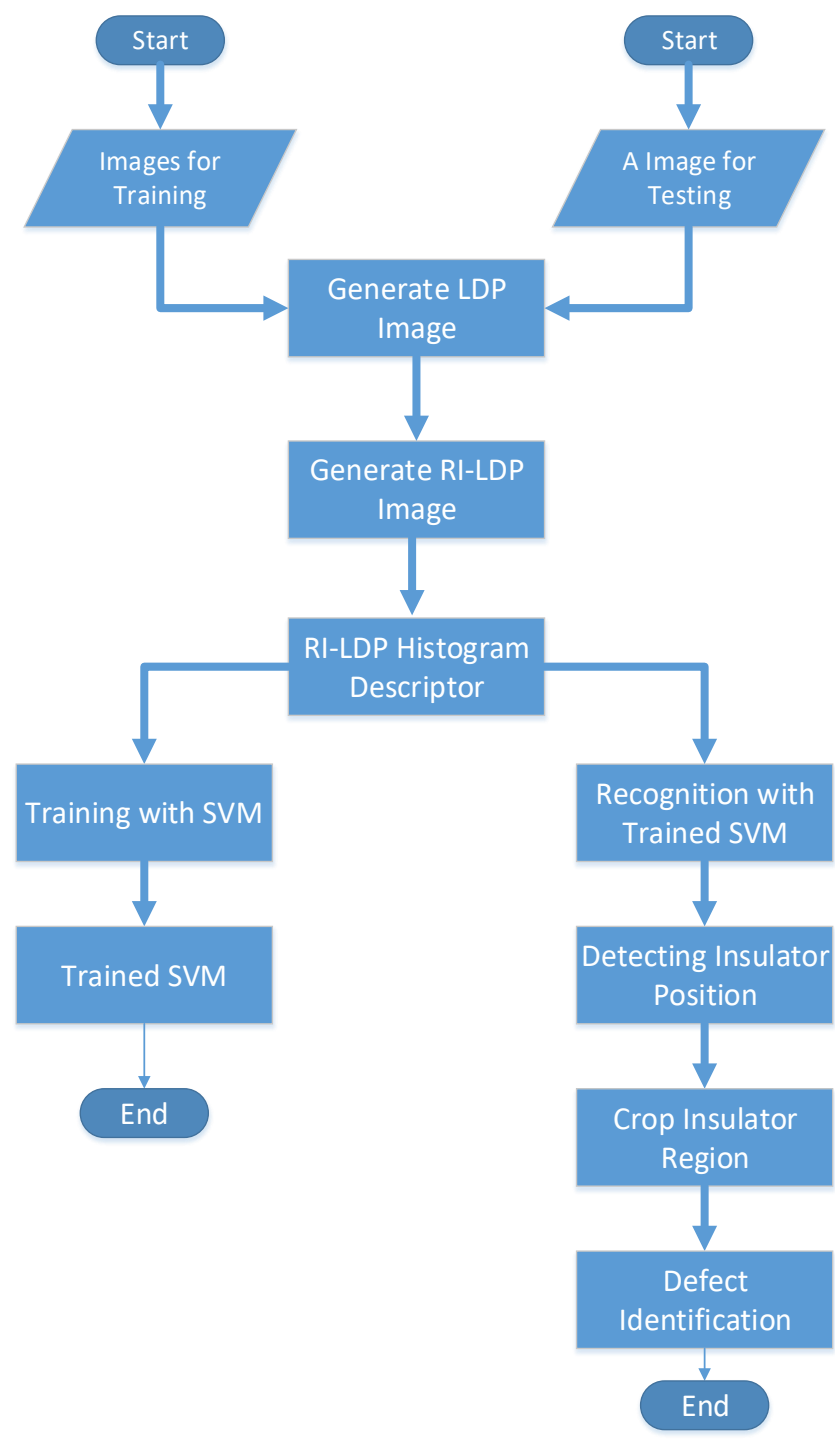

Fig. 2. Flowcharts of the proposed rotation invariant local directional based insulator detection and defect identification.

\section{INSULATOR DETECTION USING ROTATION INVARIANT LOCAL DIRECTIONAL PATTERN (RI-LDP)}

We can locate power line insulators in different size, shape, and/or texture. However there are also some trivialities. Every insulator contains a repeating patterned called cap. Number of caps may vary significantly which made the insulator length variable. Insulators are made of ceramic and/or glass which make sure low textured appearance. However, these low textured insulators mostly appear in clutter background which made it difficult the recognition. The aspect ratios of insulators vary in a certain range as long as the images are taken within tolerable viewing angle. All these properties may entice researcher to see the insulator detection problem as a generalize object detection using local texture feature [13]. However, in reality the insulators appear in varying orientation which makes the detection process complex. The rotation variation can be handled by explicitly normalizing the rotated object or using a rotation invariant feature. In this work, a 
rotation invariant local featured named rotation invariant local directional pattern (RI-LDP) is proposed which enables the detector to detect insulator caps even if those are not in same orientation. However, this rotation invariance is not sufficient to detect the whole string of insulator caps as a single insulator. Therefore, we propose a novel post processing method which finally combines series of insulator caps as a single insulator. The overall step of the proposed method is shown in Fig. 2. In the following sub-sections, the basic LDP code generation process is briefly described, and then the proposed rotation invariant (RI-LDP) is explained in details.

$$
\begin{aligned}
& {\left[\begin{array}{ccc}
-3 & -3 & 5 \\
-3 & 0 & 5 \\
-3 & -3 & 5
\end{array}\right] \quad\left[\begin{array}{ccc}
-3 & 5 & 5 \\
-3 & 0 & 5 \\
-3 & -3 & -3
\end{array}\right] \quad\left[\begin{array}{ccc}
5 & 5 & 5 \\
-3 & 0 & -3 \\
-3 & -3 & -3
\end{array}\right] \quad\left[\begin{array}{ccc}
5 & 5 & -3 \\
5 & 0 & -3 \\
-3 & -3 & -3
\end{array}\right]} \\
& \text { East } M_{0} \quad \text { North East } M_{1} \quad \text { North } M_{2} \quad \text { North West } M_{3} \\
& {\left[\begin{array}{ccc}
5 & -3 & -3 \\
5 & 0 & -3 \\
5 & -3 & -3
\end{array}\right] \quad\left[\begin{array}{ccc}
-3 & -3 & -3 \\
5 & 0 & -3 \\
5 & 5 & -3
\end{array}\right] \quad\left[\begin{array}{ccc}
-3 & -3 & -3 \\
-3 & 0 & -3 \\
5 & 5 & 5
\end{array}\right] \quad\left[\begin{array}{ccc}
-3 & -3 & -3 \\
-3 & 0 & 5 \\
-3 & 5 & 5
\end{array}\right]} \\
& \text { West } M_{4} \quad \text { South West } M_{5} \quad \text { South } M_{6} \quad \text { South East } M_{7}
\end{aligned}
$$

Fig. 3. Eight different Kirsch edge masks.

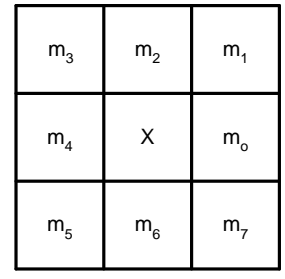

(a)

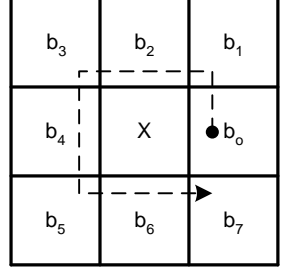

(b)
Fig. 4. (a) Eight directional edge response positions; (b) LDP binary bit positions.

\section{A. Local Directional Pattern (LDP)}

Local Directional Patten (LDP) is a local texture pattern which efficiently describes spatial structure of a tiny image patch. Typically, a local texture is calculated by analyzing image pixel value and/or gradient direction of a tiny image region. However, LDP utilizes the gradient magnitude of different direction and by analyzing all directional responses generates an eight bit binary number to describe the image patch [14], [15]. One of the main strength of LDP is utilization of edge responses instead of image pixel as edge responses are typically more robust in adverse imaging situation. Therefore, local image primitive like dark pot, corner, junction, edge, bright spot etc. can be encoded by LDP with lesser influence of external impurity. LDP can be generated for any pixel by analyzing a $3 \times 3$ images region centering at that code pixel. For this, eight directional edge responses values $\left\{m_{i}\right\}, i=0,1, \ldots, 7$ are computed by Kirsch masks $M_{i}$ in eight different orientations centered on its position. The eight different masks are shown in Fig. 3.

The relative magnitudes of response values carry noteworthy information. The higher magnitude in a particular direction ensures prominent texture in that direction. However, the relatively lower magnitudes also carry some important information. Therefore, we are interested to know the $k$ most prominent directions in order to generate the LDP. Here, the top $k$ directional bit responses $b_{i}$ are set to 1 . The remaining

\begin{tabular}{|c|c|c|c|c|c|c|c|c|c|c|}
\hline 85 & 32 & 26 & \multirow{2}{*}{$\mathrm{M}_{\mathrm{i}}$} & 313 & 97 & 503 & \multirow{2}{*}{$\stackrel{\mathrm{m}_{\mathrm{k}}}{\longrightarrow}$} & 0 & 0 & 1 \\
\hline 53 & 50 & 10 & & 537 & $x$ & 393 & & 1 & $x$ & 1 \\
\hline 60 & 38 & 45 & & 161 & 97 & 161 & & 0 & 0 & 0 \\
\hline
\end{tabular}
(8-k) bits of 8-bit LDP pattern is set to 0. Finally, the LDP code is derived by (1). Fig. 4 shows the mask response and LDP bit positions, and Fig. 5 shows an exemplary LDP code with $k=3$.

LDP Binary Code $=00010011$

\begin{tabular}{|c|c|c|c|c|c|c|}
\hline 26 & 10 & 45 & \multirow{2}{*}{$\mathrm{M}_{\mathrm{i}}$} & 503 & 393 & 161 \\
\hline 32 & 50 & 38 & & 97 & $x$ & 97 \\
\hline 85 & 53 & 60 & & 313 & 537 & 161 \\
\hline
\end{tabular}

LDP Decimal Code $=19$

Fig. 5. Example of LDP code generation with $\mathrm{k}=3$.

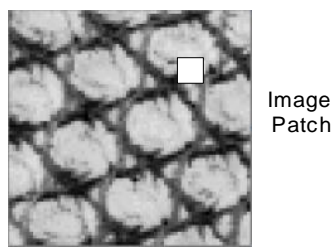

\begin{tabular}{|c|c|c|c|c|c|c|}
\hline 85 & 32 & 26 & \multirow[b]{2}{*}{$\mathrm{M}_{\mathrm{i}}$} & 313 & 97 & 503 \\
\hline 53 & 50 & 10 & & 537 & $x$ & 393 \\
\hline 60 & 38 & 45 & & 161 & 97 & 161 \\
\hline
\end{tabular}

(a)

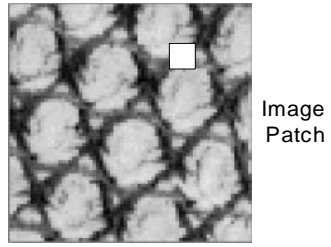

(b)

Fig. 6. Modified edge responses value after rotation of the image. (a) Original image along with eight edge response values, (b) Rotated image along with changed edge responses values.

$L D P_{k}=\sum_{i=0}^{7} b_{i}\left(m_{i}-m_{k}\right) \times 2^{i}$

$b_{i}(a)= \begin{cases}1 & a \geq 0 \\ 0 & a<0\end{cases}$

where, $m_{k}$ is the $k$-th highest value among all eight directional response values. LDP texture code provides similar pattern in adverse imaging situation like change in illumination and/or noise presence [16] because inherently directional response magnitudes are more stable than intensity values.

LDP based descriptor is calculated after computing LDP code for each pixel $(r, c)$ of the input image $I$ of size $M \times N$. LDP descriptor generation process is motivated by other texture based feature descriptor where histogram of individual feature plays the role of image descriptor [17], [18]. In accordance, we also generate a LDP histogram $H$ using for describing that image. The equation of this histogram generation is shown with (3) and (4).

$$
H(\tau)=\sum_{r=1}^{M} \sum_{c=1}^{N} f\left(L D P_{k}(r, c), \tau\right) \text {. }
$$


$f(a, \tau)=\left\{\begin{array}{cc}1 & a=\tau \\ 0 & \text { otherwise }\end{array}\right.$

where, $\tau$ is the all possible LDP code. The number of possible LDP code varies as our choice of $k$ value and can be expressed by $C_{k}^{8}=\frac{8 !}{k !(8-k) !}$.

\section{B. Rotation Invariant Local Directional Pattern (RI-LDP)}

Change in rotation appearance of an image affects the pixel value of the image. Though the spatial distribution of image pixels is modified, the relative appearance of pixels is not affected by mere rotation (see Fig. 6). The image clearly exhibits that the edge direction of the object is altered due to alteration of the image orientation. Consequently, direction of highest gradient magnitude will be modified and that will lead to a completely different LDP code. As the content of the image is same but appeared in different orientation, we require a steady LDP code to achieve rotation invariant detection. For achieving this, we analyzed the gradient magnitude of all directions and found that though the direction of highest magnitude changes, but the relative position of other lower rank magnitude with highest one is unchanged. This trend is explained using a small image patch shown in Fig. 6. In Fig. 6(a), it shows an image along with eight directional gradient magnitudes. This image is rotated by $90^{\circ}$ in a counterclockwise direction and modified gradient magnitudes in Fig. 6(b). By observing these two set of gradient magnitude, we found that the relative position of gradient magnitude is preserved, it just shifted by the corresponding image rotation value. In this example it shifted two places in rightward direction which agreed $90^{\circ}$ clockwise rotations. In addition to that, it is well known fact that image rotation in spatial domain is equivalent to circular shift in feature vector [19]. Based on these observations we proposed a simple method for achieving the rotation invariant LDP code. The proposed method performs the circular shift operation to the original LDP code until the bit representing the most prominent edge response is aligned with the least significant bit $b_{0}$ as shown in Fig. 7.

For example, if the 8 directional responses of a pixel is given by the set $\{3,5,2,2,8,4,3,2\}$, which stars with $\mathrm{m}_{0}$, then the original LDP code is 00110010 . The rotation invariant code for the pixel can be obtained by shifting the directional response as $\{8,4,3,2,3,5,2,2\}$. The resulting code is 00100011. This rotation normalization method tries to evaluate the objects with different rotational appearance by aligning the inherent texture pattern along with the most prominent texture property and then compare. This rotation invariant LDP code which is denoted by RI-LDP can be generated with (5).

$$
\text { RI-LDP }=\text { ROR(LDP, } \mathrm{d}-1)
$$

where $\boldsymbol{d}$ is the bit position of the strongest edge response and ROR defined circular shift of the bit pattern.

\begin{tabular}{|c|c|c|c|c|c|c|}
\hline 26 & 10 & 45 & \multirow{2}{*}{$M_{i}$} & 503 & 393 & 161 \\
\hline 32 & 50 & 38 & & 97 & $x$ & 97 \\
\hline 85 & 53 & 60 & & 313 & $\underline{537}$ & 161 \\
\hline & $\checkmark$ & & & & & \\
\hline$b_{5}$ & $\mathrm{~b}_{4}$ & $b_{3}$ & & 1 & 1 & 0 \\
\hline$b_{6} \mid$ & $x$ & $b_{2}$ & & 0 & $x$ & 0 \\
\hline$b_{7}$ & $\overrightarrow{b_{0}}$ & $b_{1}$ & & 0 & 1 & 0 \\
\hline
\end{tabular}

Rotation Invariant LDP Binary Code $=00110001$

Rotation Invariant LDP Decimal Code $=49$

Fig. 7. Steps of rotation invariant LDP code generation.

The LDP operator can produces at most $C_{k}^{8}$ different code, because out of eight bit data $k$ bit must be set 1 . However, when circular shift on the LDP code is applied, the left most bit certainly become 1 . Aftermath, out of remaining seven bit data $(k-1)$ bit need be set 1 . That's why number of possible rotation invariant LDP code reduces to $C_{k-1}^{7}$. In consequence rotation invariant LDP descriptor will be a histogram with $C_{k-1}^{7}$ bins in comparison of $C_{k}^{8}$ bin in original LDP. This LDP histogram based descriptor is then used to classify between insulator region and non-insulator region of an image within a sliding window framework. The two class classification is carried out using support vector machine (SVM).

\section{Support Vector Machine (SVM)}

Support vector machine (SVM) is one of the most popular machine learning techniques. SVM which is proposed by Vapnik et al. [20] is shown effectiveness as supervised pattern recognition. During classification, SVM try to separate a given set of labeled data with the best hyperplane by transforming it into higher dimension. SVM choose the hyperplane when the distance from hyperplane to the closest data point of each class is maximized. Feature vector for any image processing problem is non-linear; hence SVM apply complex but easy to implementable kernel without using potentially infinite dimensional feature vector. The hyperplane found by the SVM in the high dimensional feature space corresponds to a nonlinear boundary in the input space.

The choice of appropriate kernel and different parameter associate to the selected kernel is very critical during classification performance using SVM. In literature, we found that linear, polynomial and radial basis function (RBF) kernels are the most frequently used during image based object detection. The choice of inappropriate kernel parameter can 
leads to very poor classification accuracy. Hence grid-search approach is applied for selecting the parameters [21]. The parameter of specific kernel that produces best classification accuracy is picked.

Typical SVM based object detection requires number of training samples grouped into two sets - one is positive sample and other one is negative sample. These two sets are used to train a binary classifier which can subsequently predict where an unknown sample belongs. However, to make the training computationally viable, only a subset of possible sample data is used. D. King [22] proposed Maximum Margin Object Detection (MMOD) method which tries to optimize the classifier using all the candidate windows available in the image. In this work, dlib's [23] structural SVM based algorithm is used which enables us to train on all the subwindows in every image.

\section{INSULATOR DEFECT ANALYSIS}

We proposed an insulator defect analysis system which can evaluate detected insulator region for identifying the potential defect. There are different kinds of defects which deteriorate the effectiveness of the insulators. Some of those defects modify the electrical property of the insulator rather than appearance. These types of defect are out of our vision-based identification system's scope. Rather we only focused on the defects which alter the appearance of the insulators like change the color, shape and/or texture. Some of those defects affect core region while some others affect in the cap region. In addition to that sometimes defects may appear in some caps while others remain in good condition. Hence, each cap should be individually segmented from the core region. After that, we can analyze each caps or core region for identifying the defects. Hence, the defect analysis work can be separated into two parts: 1) insulator partitioning, 2) defect identification.

\section{A. Insulator Partitioning}

Individual caps are detected by detecting elliptical regions on the detected insulator region followed by clustering based on orientation and size of the ellipses. At first, elliptical shaped regions in the insulator are detected by a method proposed by Fornaciari [24]. All the detected elliptical shaped arcs are labeled into four groups and estimate the ellipse parameters using the decomposed parameter space. At first, edge pixels are classified in two main directions (i.e., positive and negative) according to their gradient phases. Edge pixels with the same gradient phases are grouped together and classified as an arc according to their convexity.

This method is capable to detect those ellipses whose arcs are visible and can be detected in at least three quadrants. Consequently, this method looks for combinations of three arcs, called triplets, each belonging to a different quadrant. A selected triplet forms a candidate ellipse. As from the triplet information, we may already know its center; we estimate the remaining three parameters in a decomposed Hough space requiring three $1 \mathrm{D}$ accumulators. Candidate ellipses are then validated according to the fitness of the estimation with the actual edge pixels.
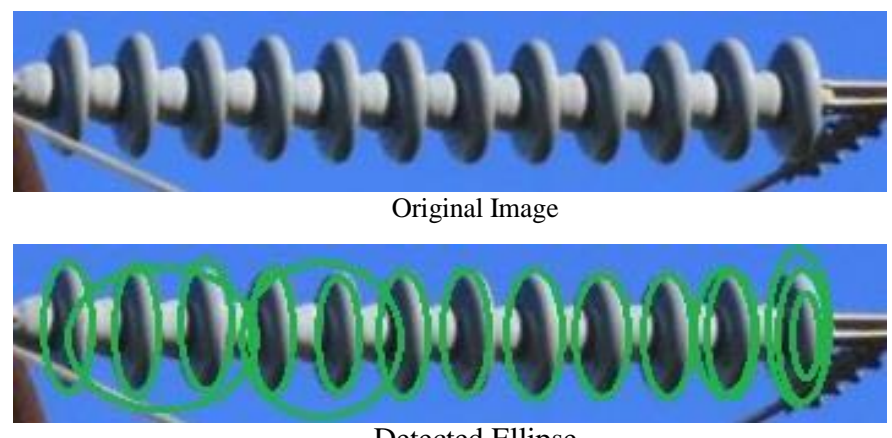

Detected Ellipse

Fig. 8. Detected ellipse from the insulator region.

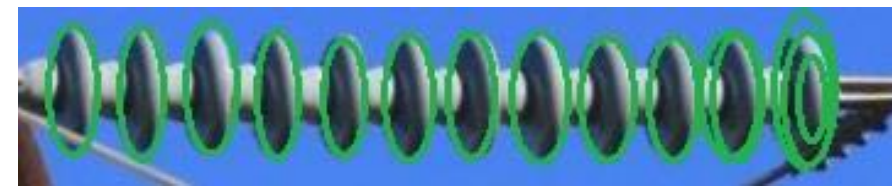

Fig. 9. Detected ellipse after selecting ellipses of largest group.

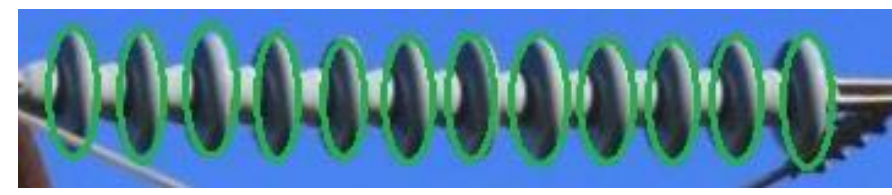

Fig. 10. Separately detected each insulator.

However, due to possible clutter in the image some non-cap region can be detected as an ellipse (see Fig. 8). We have to eliminate those non-caps region and keep only the true cap regions. To achieve this, we cluster the ellipses based on their orientation and size and ellipses belongs to the largest cluster are retained. Sometimes, multiple ellipses can be detected around a single cap as shown in Fig. 9. We keep only one ellipse if there are multiple overlap ellipses depending on the ellipse parameters of the other ellipses of the same group. The result after this step is shown in Fig. 10.

\section{B. Defect Identification}

After partitioning insulator, each cap is analyzed for defect identification. A number of different defects may appear in the insulators. In this work, we attempt to identify defects which alter appearance i.e. color, shape and/or texture of the insulator. Defects which change only physical property of the insulator but not appearance is out of scope this research work. Therefore, in this work we consider the following defects: 1) whitening; 2) aligatoring effect; 3) bullet damage; 4) contamination; and 5) crack.

All of the above mentioned defects change the cap's surface area which is normally smooth. Therefore, we can identify whether any cap is altered by the analyzing local texture of the cap's region. In this respect, we calculate the LDP histogram from each of the cap region for identifying whether cap is affected by any of these defects. This calculated LDP histogram is then feed into a multi-class classifier for identify specific defect. However, due to lack of availability of defected sample, sophisticated classifier like SVM or CNN is not 
utilized rather simple nearest-neighbor classifier is used in classify the defect type. As our proposed LDP descriptor is histogram based feature, we need to calculate similarity or dissimilarity between two histograms. For this we can choose any one from Histogram intersection, Log-likelihood statistics and/or Chi square statistics $\left(\mathrm{x}^{2}\right)$. In this work Chi square statistics is used for comparing two histograms.

$$
\chi^{2}(S, M)=\sum_{i} \frac{\left(S_{i}-M_{i}\right)^{2}}{\left(S_{i}+M_{i}\right)}
$$

\section{EXPERIMENTAL RESULT}

In this section, insulator detection and defect identification results are presented and discussed. As there is no publicly available dataset for insulator detection, we develop our own evaluation dataset with two different image resolutions. Lowresolution dataset contains 298 images where in total 1470 insulators appeared in those images. However, the highresolution dataset contains 395 images $(2592 \times 1944$ pixels $)$ with 722 labeled insulators. The segmented ground-truth information is generated by manually tagging the insulators.

\section{A. Result of Insulator Detection}

The performance of the insulator detector is evaluated by finding detection accuracy of the marked insulators. As insulator appears in arbitrary orientation in the dataset, each insulator is marked by a rotated rectangle. To decide whether detected window is correct detection or not, well-known Pascal scores [25] has been used. Pascal score is calculated from the overlap of our generated bounding box $\mathbf{B}_{\mathbf{c l}}$ to the ground-truth

$\mathbf{B}_{\text {gt }}$ by $P\left(B_{c l}, B_{g t}\right)=\frac{\operatorname{area}\left(B_{c l} \cap B_{g t}\right)}{\operatorname{area}\left(B_{c l} \cup B_{g t}\right)}$. An object is considered detected if $P\left(B_{c l}, B_{g t}\right)>0.5$

a) Detection in Low-Resolution Images: Our objective is to maximize the number of correct detections and minimize the number of false detections. The two used evaluation metrics are precision, the fraction of correct detections to the total number of detections made by our detector, and recall, the fraction of correctly detected objects to the number of annotated objects. Our detector provides a score for each detection, and with average detection score, we achieved recall rate $51.29 \%$ with $81.82 \%$ precision in low-resolution image dataset. But in low-resolution images, our objective is detecting correct insulators more even if it provides some false detection. Because those false alarms can be eliminated by later steps in high resolution. By lowering detection threshold, we can relax the detection criteria which in turn increase recall rate in the expense of precision. The result is shown in Table III by varying detection threshold. With detection threshold -0.3 , our system can detect more than $80 \%$ of insulators from low-resolution images. Some of the insulators which are not detected appeared close to already detected insulators. Therefore, when the second camera zoomed in towards the detected insulators the missed insulators will also appear in high-resolution images. Consequently, those missed insulators can be detected in high-resolution.

b) Detection in High Resolution Images: In highresolution image, the objective is same as low resolution i.e., to maximize the number of correct detections and minimize the number of false detections. However in high-resolution, we cannot tolerate high false alarm as we do not have any further steps which will eliminate those. With average detection score, the proposed system achieved recall rate $95.74 \%$ with $89.94 \%$ precision. Table I shows recall and precision rate of the proposed method and other comparable method. It clearly demonstrate the suitability of the proposed method over other state of the art techniques. Table I shows that recall rate of the proposed method is just behind the method of Oberweger et al. However, the precision rate is much higher than that of Oberweger et al.

TABLE I. PRECISION AND ReCAll CURVE of Proposed Method AND OTHER METHODS

\begin{tabular}{|l|l|l|}
\hline Method & Recall & Precision \\
\hline Oberweger et al [4] & $98 \%$ & $33 \%$ \\
\hline Liao and An [5] & $91 \%$ & $87 \%$ \\
\hline Wu and An [2] & $86.47 \%$ & $85.59 \%$ \\
\hline Proposed Method & $95.74 \%$ & $89.94 \%$ \\
\hline
\end{tabular}

For further analyze the performance of proposed method, we compare precision recall (PR) curve. As our detector provides a score for each detection, we can vary this score to elaborate the trade-off between recall and precision metrics. Fig. 11 exhibits the precision recall curve of our method and Oberweger's method which exhibits the superiority of the proposed system.

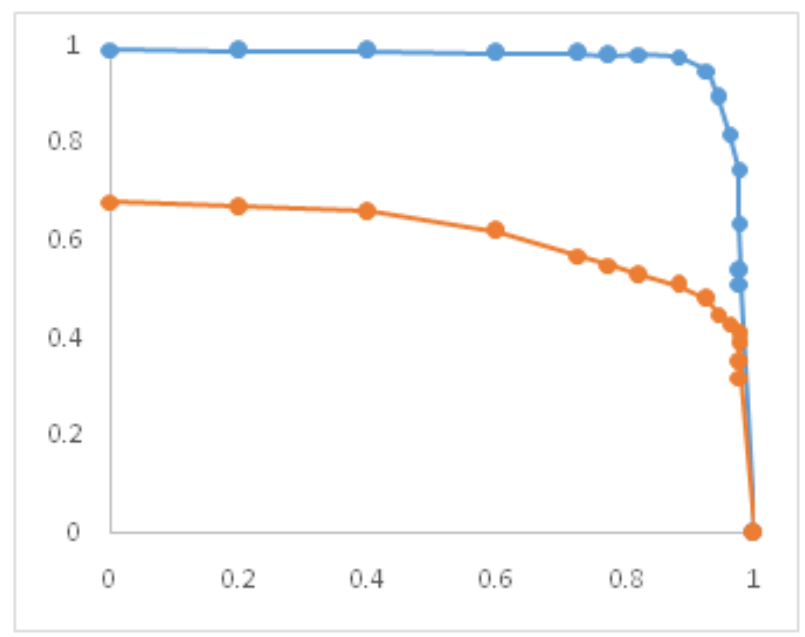

Fig. 11. Precision-recall curve for insulator detection.

By further scrutinizing the detected insulator images, we found that some of the insulators are considered as not detected due to low overlap value. In our evaluation, the required Pascal criterion was 0.5 which is very strict due to the fact that we use rotated bounding boxes. However the originally Pascal criterion is intended for axis aligned bounding box. Subjectively speaking, an overlap score of 0.5 fits the insulator very well, whereas a lower threshold might be good enough for visually consider as a true detection. Therefore, lowering 
Pascal criterion may improve our detection performance in expense of some loss in precision and the result with varying overlap threshold is shown in Table II.

TABLE II. InSUlator DETECTION RESUlt WITH VARYING PASCAL OVERLAP THRESHOLD

\begin{tabular}{|l|l|l|}
\hline Overlap Threshold & Recall & Precision \\
\hline 0.50 & $95.74 \%$ & $89.94 \%$ \\
\hline 0.40 & $94.79 \%$ & $89.39 \%$ \\
\hline 0.30 & $94.94 \%$ & $89.22 \%$ \\
\hline
\end{tabular}

\section{B. Result of Insulator Defect Analysis}

A proper insulator partitioning is essential for effective defect analysis. By insulator portioning, we mean separation of each cap from one another so that we can analyze each cap independently. Therefore we first evaluate the insulator partitioning and then the defect identification itself.

1) Result of Insulator Partitioning: For the evaluation of insulator portioning, we compare number of caps correctly segmented $\left(\mathbf{N}_{\mathbf{s g}}\right)$ by proposed method with ground truth number of visible caps $\left(\mathbf{N}_{\mathbf{g t}}\right)$ in the insulator image. As evaluation criterion of correct cap segmentation, we compare ground truth cap area with segmented cap area with Pascal score. If Pascal score is above 0.8 then we consider the cap as a correct detection. Finally, an insulator is considered as correctly partitioned if the following condition is true.

$$
\operatorname{abs}\left(N_{s g}-N_{g t}\right)<=1
$$

The appearance of insulators varies a lot due to image viewing angle. The appearance of caps can be near frontal to almost side view. We group the insulator images in three categories depending on the viewing angle of the image.

- Group1: Viewing angle of the insulators are almost frontal.

- Group 2: Viewing angle of the insulators varies from $\sim 10^{\circ}$ to $\sim 50^{\circ}$.

- Group 3: Viewing angle of the insulators are more than $50^{\circ}$.

It is almost impossible to segment the caps from insulator of group1 as caps are not individually visible. However, the core part is visible in this orientation. So we detect core region when images are captured in this orientation. When the viewing angle increased from near frontal, the cap region becomes separable. Therefore, we partition each insulator of these two groups and result of partitioning is shown in Table III.

TABLE III. PERFORMANCE OF INSULATOR PARTITIONING

\begin{tabular}{|l|l|l|l|}
\hline Group No & $\begin{array}{l}\text { Number of } \\
\text { Insulator } \\
\text { Images }\end{array}$ & $\begin{array}{l}\text { Number of Insulator } \\
\text { Correctly Partitioned }\end{array}$ & $\begin{array}{l}\text { Partition } \\
\text { Accuracy }\end{array}$ \\
\hline Group 2 & 76 & 73 & $96.05 \%$ \\
\hline Group 3 & 17 & 8 & $47.05 \%$ \\
\hline
\end{tabular}

2) Result of Defect Identification: To the best of our knowledge, there is currently no publicly available dataset for insulator defect identification. Hence, we use our own insulator database to analyze the defect identification performance. There are five defected insulator images for each type of defect. Furthermore, we add another 20 non-defected images to the database. Accordingly, there are 45 images in our defect identification database with six caps in each insulator making total $6 \times 45=270$ insulator cap. Out of these 270 caps, $20 \times 6=120$ caps are in good condition and other 250 $(25 \times 6)$ contains one of those five defects. The overall defect identification result is shown in Table IV. So far, we have discussed the average defect identification accuracy of five different defects. To get a better picture of the identification accuracy of individual defect types, the confusion matrix is shown in Table V.

TABLE IV. PERFORMANCE OF INSUlator DEFECT IDENTIFICATION

\begin{tabular}{|l|l|l|}
\hline Number of Caps & $\begin{array}{l}\text { Number of Caps } \\
\text { correctly classified }\end{array}$ & $\begin{array}{l}\text { Defect identification } \\
\text { accuracy }\end{array}$ \\
\hline 270 & 244 & $90.37 \%$ \\
\hline
\end{tabular}

TABLE V. CONFUSION MATRIX OF 6-Class DEFECT ClassificATION

\begin{tabular}{|l|l|l|l|l|l|l|}
\hline & White. & Alliga. & Bullet. & Contam. & Crack & $\begin{array}{l}\text { No } \\
\text { Def. }\end{array}$ \\
\hline Whitening & 28 & 0 & 0 & 0 & 1 & 1 \\
\hline Alligatoring & 2 & 26 & 0 & 0 & 2 & 0 \\
\hline Bullet & 0 & 1 & 25 & 0 & 3 & 1 \\
\hline Contamination & 0 & 1 & 0 & 28 & 0 & 1 \\
\hline Crack & 0 & 2 & 1 & 0 & 27 & 0 \\
\hline No Defect & 1 & 0 & 0 & 4 & 5 & 110 \\
\hline
\end{tabular}

\section{CONCLUSION}

In this work, we have presented a rotation invariant local directional feature showing encoding scheme for representing texture of insulator image. The proposed rotation invariant local directional pattern (RI-LDP) feature shows efficacy in detecting rotated insulator. We also show the effectiveness of the proposed method in the low-resolution image which allows us coarse to fine detection approach. Experimental results illustrate that suitability of the proposed method over other state-of-the-art methods. After successful detection and segmentation of the insulators from images with the cluttered background, their condition is analyzed by a gradient based feature named local directional pattern (LDP). The insulators are partitioned into the individual cap for better defect identification. The results of defect identification show that almost all defective insulators can be separated from the good insulator. Thus the proposed insulator analysis system would improve the reliability of power supply and the requirement of technical manpower can also be substantially reduced as nontechnical persons can be employed for acquiring images and feeding to the system for analysis.

\section{REFERENCES}

[1] W. G. Li, G. Ye, F. Huang, S. Wang, and W. Z. Chang, "Recognition of insulator based on developed MPEG-7 texture feature," in Proc. IEEE ICISP, pp. 265-268, 2010.

[2] Q. Wu and J. An, "An active contour model based on texture distribution for extracting inhomogeneous insulators from aerial images", IEEE Trans. Geosci. Remote Sens., vol. 52, no. 6, pp. 3613-3626, 2014.

[3] X. Wang and Y. Zhang, "Insulator Identification from Aerial Images Using Support Vector Machine with Background Suppression” in Proc. 
International Conference on Unmanned Aircraft Systems (ICUAS) June 7-10, pp. $892-897,2016$.

[4] M. Oberweger, A. Wendel, and H. Bischof, "Visual recognition and fault detection for power line insulators," in Proc. 19th Computer Vision Winter Workshop, pp. 1-8, 2014.

[5] S. Liao, J. An, "A robust insulator detection algorithm based on local features and spatial orders for aerial images," IEEE Geosci. Remote Sens. Lett. vol. 12, no. 5, pp. 963-967, 2015.

[6] Y. Zhai, Y. Wu, H. Chen, and X. Zhao, "A method of insulator detection from aerial images," Sensors \& Transducers, vol. 177, no. 8, 2014.

[7] B. Li, D. Wu, Y. Cong, Y. Xia, and Y. Tang, "A method of insulator detection from video sequence," in 2012 International Symposium on Information Science and Engineering (ISISE), pp. 386-389, 2012.

[8] Y. Tiantian, Y. Guodong, Y. Junzhi, "Feature Fusion Based Insulator Detection for Aerial Inspection", Proceedings of the 36th Chinese Control Conference, July 26-28, 2017, Dalian, China

[9] Z. Zhao, X. Fan, G. Xu, L. Zhang, Y. Qi, and K. Zhang, "Aggregating Deep Convolutional Feature Maps for Insulator Detection in Infrared Images" IEEE Access, vol. 5, 2017.

[10] T. Jabid and M.Z. Uddin, "Rotation invariant power line insulator detection using local directional pattern and support vector machine", IEEE Conference on Innovations in Science Engineering and Technology (ICISET), 2016.

[11] V.S. Murthy, K. Tarakanath, D.K. Mohanta, S. Gupta, "Insulator condition analysis for overhead distribution lines using combined wavelet and support vector machine (SVM)," IEEE Transactions on Dielectrics and Electrical Insulation, vol. 17, no. 1, pp. 89-99, 2010

[12] V. S. Murthy, S. Gupta and D. K. Mohanta, "Digital image processing approach using combined wavelet hidden Markov model for well-being analysis of insulators", IET Image Process., vol. 5, no. 2, pp. 171-183, 2011.

[13] D.A. Lisin, M.A. Mattar, M.B. Blaschko, E.G. Learned-Miller, M.C. Benfield, "Combining local and global image features for object class recognition," in Workshop on Learning in Computer Vision and Pattern Recognition at IEEE CVPR, 2005.
[14] T. Jabid, M. H. Kabir, and O. S. Chae, "Robust Facial Expression Recognition based on Local Directional Pattern," ETRI Journal, vol. 32, no. 5, October, 2010. Pp. 784-794.

[15] T. Jabid, M. H. Kabir, and O. S. Chae, "Local Directional Pattern (LDP) for Face Recognition," IEEE International Conference on Consumer Electronics, January 2010.

[16] T Jabid, MH Kabir, O Chae, "Local Directional Pattern (LDP) A Robust Image Descriptor for Object Recognition", Proceedings of the IEEE Advanced Video and Signal Based Surveillance (AVSS), pp. 482-487, August 29-September 1.

[17] D. Lowe, "Distinctive Image Features from Scale-Invariant Keypoints", Int'1 J. Computer Vision, vol. 2, no. 60, pp. 91-110, 2004.

[18] N. Dalal, B. Triggs, "Histograms of Oriented Gradients for Human Detection", Proc. IEEE Conf. Computer Vision and Pattern Recognition, 2005.

[19] D. Zhang, A. Wong, M. Indrawan, and G. Lu, "Content-based image retrievalusing gabor texture features," in IEEE Transactions PAMI, 2000, pp. 13-15.

[20] C. Cortes and V. Vapnik, "Support vector networks", Machine Learning," vol. 20, no. 3, pp. 273-297, 1995.

[21] C. -W. Hsu and C. -J. Lin, "A comparison on methods for multiclass support vector machines," IEEE Trans. Neural Networks, vol. 13, no. 2, 2002, pp. 415-425.

[22] D. E. King, "Max-margin object detection", CoRR, 2015

[23] D. E. King, "Dlib-ml: A machine learning toolkit", Journal of Machine Learning Research, pp. 1755-1758, 2009

[24] M. Fornaciari, A. Prati, and R. Cucchiara, "A fast and effective ellipse detector for embedded vision applications," Pattern Recognition, vol. 47, no. 11, pp. 3693-3708, 2014.

[25] M. Everingham, L. J. V. Gool, C. K. I. Williams, J. M. Winn, and A. Zisserman, "The Pascal Visual Object Classes (VOC) Challenge", IJCV, vol. 88, no. 2, pp. 303-338, 2010. 